\title{
Cirrhosis and $C$. difficile: A Deadly Duo?
}

\author{
Stephen M. Vindigni ${ }^{1} \cdot$ Christina M. Surawicz $^{1}$
}

Published online: 12 July 2015

(c) Springer Science+Business Media New York 2015

Patients with advanced liver disease develop complications as their disease progresses, including hepatic encephalopathy, ascites, spontaneous bacterial peritonitis (SBP), and variceal bleeding. Moreover, they have decreased life expectancy with high rates of mortality related to infection, bleeding, and multi-organ failure, including the hepatorenal syndrome. Patients with cirrhosis are also at increased risk of developing Clostridium difficile infection (CDI) with several identified risk factors including frequent hospitalizations, regular use of antibiotics for prophylaxis or treatment for SBP, proton pump inhibitor (PPI) use, and an overall immunocompromised state. In a study of the impact of CDI on inpatients with cirrhosis, Bajaj et al. [1] reported increased mortality, longer hospitalization, and higher hospitalization charges in patients with both cirrhosis and CDI compared to patients with either cirrhosis or CDI alone $(p<0.001)$.

Since patients with cirrhosis and CDI have a higher mortality, Saab and colleagues addressed the question of whether screening patients with cirrhosis on admission to the hospital, with isolation and treatment to eradicate $C$.

Disclaimer The findings and conclusions in this editorial are those of the authors and do not necessarily reflect the views of the University of Washington. All authors declare no support from any organization for the submitted work and no financial relationships with any organizations that might have an interest in the submitted work. The authors declare that they have no competing interests.

\section{Stephen M. Vindigni}

vindigni@uw.edu

1 Division of Gastroenterology, Department of Medicine, University of Washington School of Medicine, 1959 NE Pacific Street, Box 356424, Seattle, WA 98195-6424, USA difficile, will decrease mortality and healthcare costs [2]. In this issue of Digestive Diseases and Sciences, they published the results of a two-arm study using a Markov decision analysis model to compare this strategy with testing and treating only symptomatic patients with diarrhea.

The authors report that the screen and treat strategy improved healthcare outcomes with a significant cost-savings and decrease in healthcare utilization. They projected a decrease in CDI-related mortality and a 3.54-fold reduced cost in the screening group. Their interpretation and resultant recommendation is to implement a $C$. difficile screening program targeted at hospitalized patients with cirrhosis.

While this recommendation is interesting, it contradicts important points documented in two current published guidelines, that is, (1) test-only diarrheal stools and (2) do not treat $C$. difficile carriers [3, 4]. We shall now discuss these points in more detail.

\section{Only Test for $C$. difficile in Diarrheal Stools}

Rates of $C$. difficile colonization in asymptomatic patients are reported to be $0.6-21 \%$, although few assessments of toxigenic versus non-toxigenic strains were reported [5-8]. Risk factors for colonization include prior hospitalization, prior CDI, corticosteroid use, prior antibiotics, and endstage renal disease requiring hemodialysis, although heterogeneity exists among studies. A more recent study by Kong et al. [6] reported a $4.05 \%$ prevalence, but more importantly, of these colonized patients, most $(79.4 \%$, $n=166$ of 212 isolates) had non-toxigenic strains of $C$. difficile, suggesting that only a small portion of patients may harbor a pathogenic strain of $C$. difficile and thus be 
susceptible to CDI progression. Few studies have examined colonization among patients with cirrhosis.

With the concern that asymptomatic, colonized patients with cirrhosis may be at risk for CDI development, Saab et al. support their screening recommendation by referencing papers by Zacharioudakis and Lanzas [9, 10]. Zacharioudakis et al. [9] performed a systematic review and meta-analysis assessing the prevalence of $C$. difficile colonization and the risk of developing infection; they reported an $8.1 \%$ colonization prevalence among asymptomatic patients when first hospitalized, with a 5.9 times higher risk of CDI development compared to non-colonized patients during hospitalization. While these rates appear substantial, only $8.1 \%$ were colonized among all screened admits $(n=8725)$; of this smaller cohort (based on eight studies), $21.8 \%$ developed CDI. Acknowledging calculation biases, this would equate to $\sim 154$ patients, or $\sim 1.8 \%$ of the entire screened admitted population. Previously, hospitalized patients were $63 \%$ more likely to be colonized [9]. Alternatively, Lanzas and Dubberke [10] used a model to determine the effectiveness of a $C$. difficile screening program among asymptomatic carriers at hospital admission; the model suggests that screening would reduce new colonizations and hospital-onset CDI cases. Neither of these studies focuses on patients with cirrhosis; therefore, it is difficult to generalize the results to this specific population.

Based on the above evidence base, we acknowledge that some patients will be colonized with a toxigenic strain of C. difficile on hospital admission; however, there are two concerns that arise:

(a) Will these patients progress to develop active infection? As discussed in the above studies, most colonized patients will not become infected.

(b) Are these patients a source of infection and/or is there a risk of transmission to other patients in the hospital? This question is particularly important for hospitalized patients who are immunocompromised or have significant medical comorbidities and may be at increased risk of developing CDI and succumbing to its complications. Nosocomial spread of $C$. difficile has been well recognized for decades, with ample documentation that inpatients with $C$. difficile are a source of transmission to other patients, as is transient carriage of the organism on the hands of hospital personnel, confirmed using molecular methodology [11]. Given the mounting evidence that colonized patients may be a source for disease transmission, maintaining proper infection control practices for all patients and practicing sound antibiotic stewardship continue to be the cornerstones of the prevention of CDI in the hospital setting. Asymptomatic, unscreened colonized patients should not be a transmission risk, since universal precautions including strict hand hygiene (even with alcohol-based hand gels) should limit spread. This places additional responsibility on all providers to clean stethoscopes and other medical equipment when caring for patients. Furthermore, thorough environmental cleaning of patient rooms is paramount prior to admission of a new patient who may be exposed to spores remaining on hospital room surfaces. These basic preventive measures were in use as early as 1989, as illustrated in a study by McFarland et al. [12] and have continued to be indispensible practices today.

\section{C. difficile Should Not Be Treated in Carriers}

While Dr. Saab offers an impressive and provocative decision analysis model with a resultant recommendation for targeted $C$. difficile screening, there is limited evidence to support such a strong recommendation. Even if such a targeted screening program were developed, guidelines would then need to be established regarding how to interpret and respond to the results. Independent of the significant financial cost, if every colonized $C$. difficile patient were treated with antibiotics in an effort to eradicate the bacterium, the excess risks of adverse medication reactions and the promotion of antibiotic resistance in countless patients who potentially never would have progressed to CDI must be considered. Furthermore, treatment for colonized patients is of questionable effectiveness. Johnson et al. reported that attempted eradication of asymptomatic C. difficile colonization with vancomycin or metronidazole treatment was ineffective as most patients were recolonized within 2 months of treatment [7, 13].

\section{Next Steps}

Given the lack of a strong evidence base with clinical intervention trials, we cannot recommend universal screening of asymptomatic patients, including those with cirrhosis. Instead, we would stress proper hand hygiene, limiting the use of broad-spectrum antibiotics, and only testing symptomatic individuals for CDI. This latter point may be particularly challenging in patients with cirrhosis receiving lactulose for hepatic encephalopathy as this medication is often associated with loose stools; however, 
this judgment must be left up to the inpatient care team at the time of admission. We recommend close vigilance for the development of CDI symptoms and encourage hospital laboratories to consider polymerase chain reaction (PCR) testing as a rapid means of diagnosis, even though stool culture is the currently accepted reference test [4].

We appreciate the extensive analysis performed by Saab et al., particularly the raising of this important issue as a catalyst for future discussion and research. Additional studies would be beneficial in determining the link between colonization and infection. If there is progression from colonization to CDI, we must identify the risk factors that contribute to this transition-is it the strain of $C$. difficile, the host immune system, or the use of antibiotics for SBP prophylaxis? Is there a difference depending on the etiology of cirrhosis?

Study of the intestinal microbiome is currently an exciting area of research that may provide additional information regarding this important topic. Although little is known about the microbiome in liver disease, emerging data suggest that there may be a relationship between microbial composition and liver disease. The role of the microbiome as it relates to $C$. difficile colonization and disease is quite clear, and several studies have shown prior hospitalization to be a risk factor for colonization. The reason for prior admission may be important in that prior hospitalization for an infection or severe illness (with or without antibiotic therapy) may contribute to microbiome disruption, which then increases the probability that $C$. difficile will colonize. Furthermore, study of the restoration of the microbiome with probiotics is challenged by the wide range of probiotic combinations and doses without any clear evidence of superiority among the many tested. A growing body of evidence in this area is likely to emerge in the coming years with the ultimate goal of decreasing CDI-related morbidity and mortality among patients with cirrhosis.

\section{Compliance with Ethical Standards}

Conflict of interest None.

\section{References}

1. Bajaj JS, Ananthakrishnan AN, Hafeezullah M, et al. Clostridium difficile is associated with poor outcomes in patients with cirrhosis: a national and tertiary center perspective. Am J Gastroenterol. 2010;105:106-113.

2. Saab S, Alper T, Sernas E, Pruthi P, Alper MA, Sundaram V. Hospitalized patients with cirrhosis should be screened for Clostridium difficile colitis. Dig Dis Sci. (Epub ahead of print). doi:10.1007/s10620-015-3707-8.

3. Cohen SH, Gerding DN, Johnson S, et al. Clinical practice guidelines for Clostridium difficile infection in adults: 2010 update by the society for healthcare epidemiology of America (SHEA) and the infectious diseases society of America (IDSA). Infect Control Hosp Epidemiol. 2010;31:431-455.

4. Surawicz CM, Brandt LJ, Binion DG, et al. Guidelines for diagnosis, treatment, and prevention of clostridium difficile infections. Am J Gastroenterol. 2013.

5. Alasmari F, Seiler SM, Hink T, Burnham CA, Dubberke ER. Prevalence and risk factors for asymptomatic Clostridium difficile carriage. Clin Infect Dis. 2014;59:216-222.

6. Kong LY, Dendukuri N, Schiller I, et al. Predictors of asymptomatic Clostridium difficile colonization on hospital admission. Am J Infect Control. 2015;43:248-253.

7. Hung YP, Lee JC, Lin HJ, et al. Clinical impact of Clostridium difficile colonization. J Microbiol Immunol Infect. 2015;48: 241-248.

8. Leekha S, Aronhalt KC, Sloan LM, Patel R, Orenstein R. Asymptomatic Clostridium difficile colonization in a tertiary care hospital: admission prevalence and risk factors. Am J Infect Control. 2013;41:390-393.

9. Zacharioudakis IM, Zervou FN, Pliakos EE, Ziakas PD, Mylonakis E. Colonization with toxinogenic $C$. difficile upon hospital admission, and risk of infection: a systematic review and metaanalysis. Am J Gastroenterol. 2015;110:381-390.

10. Lanzas C, Dubberke ER. Effectiveness of screening hospital admissions to detect asymptomatic carriers of Clostridium difficile: a modeling evaluation. Infect Control Hosp Epidemiol. 2014;35:1043-1050.

11. Galdys AL, Curry SR, Harrison LH. Asymptomatic Clostridium difficile colonization as a reservoir for Clostridium difficile infection. Expert Rev Anti Infect Ther. 2014;12:967-980.

12. McFarland LV, Mulligan ME, Kwok RY, Stamm WE. Nosocomial acquisition of Clostridium difficile infection. $N$ Engl J Med. 1989;320:204-210.

13. Johnson S, Homann SR, Bettin KM, et al. Treatment of asymptomatic Clostridium difficile carriers (fecal excretors) with vancomycin or metronidazole. A randomized, placebo-controlled trial. Ann Intern Med. 1992;117:297-302. 